\title{
Numerical Models of Accretion Disks
}

\author{
Charles F. Gammie \\ Harvard-Smithsonian Center for Astrophysics \\ 60 Garden St., MS-51, Cambridge, MA 02138 USA \\ cgammie@cfa.harvard.edu
}

\begin{abstract}
I review recent numerical studies of accretion disks, focusing on measurements of the turbulent shear stress, or $\alpha$, in the shearing box model. I conclude with a list of astronomically relevant open questions that can be settled via future numerical experiments.
\end{abstract}

\section{INTRODUCTION}

The last decade has brought a flood of cheap, fast workstations and easy access to supercomputers to the astronomical community. For those struggling to understand complicated phenomena like the active nucleus of NGC 4258, with its beautifully precise maser spots orbiting in a thin, warped disk, these machines seem to offer the seductive possibility of creating a detailed and comprehensive simulation. One could imagine that by including all the relevant physics, such a simulation would self-consistently generate X-rays from the relativistic accretion flow in the neighborhood of the black hole (ending the debate between proponents of ADAF and disk corona models); it would warp straightaway (giving away the origin of the warp); it would include a chemical network to calculate the local abundance and excitation of water; it would perform three dimensional (3D) radiative transfer in the water maser lines; and of course, it would produce a jet. Our curiosity would be completely satisfied.

Of course, such global and physically complete simulations are mere fantasy. They are not possible now, nor will they be except in some distant, post-Moore'slaw future world. It is not even clear that they would be desirable! Even if one could determine the right initial conditions - probably impossible even in principle- the output would be so complicated (cf. [1] on spiral structure) that it would not be clear what was important.

Absent global, ab initio simulations one is reduced to solving simplified model equations that rely on reasonable but untested physical assumptions. Thus models of dwarf novae (see, e.g. [2,3]) typically evolve an azimuthally averaged and heightintegrated set of equations for the surface density $\Sigma$ : 


$$
\partial_{t} \Sigma(r, t)=\frac{2}{r} \partial_{r}\left(\frac{1}{r \Omega} \partial_{r}\left(r^{2} W_{r \phi}\right)-\frac{\tau}{\Omega}\right)-\dot{\Sigma}_{W}
$$

Here $\Sigma \equiv$ surface density, $\Omega \equiv$ rotation frequency, $\dot{\Sigma}_{W} \equiv$ mass lost per unit area in winds, $W_{r \phi} \equiv \int d z w_{r \phi} \equiv$ height-integrated shear stress, and $\tau \equiv$ direct torque per unit area- possibly supplied by a magnetohydrodynamic (MHD) wind or by the tidal field of the secondary. Equation (1) is in a sense fundamental; it is derived from the angular momentum and continuity equations in the limit that the disk is thin.

In solving equation (1) two assumptions are almost always made. First, $\tau=$ $\dot{\Sigma}_{W}=0$. This assumption is required by our ignorance of the very difficult, global problem of disk winds; the relative importance of external torques $\tau$ and internal stresses $w_{r \phi}$ remains one of the outstanding problems of disk physics. Second,

$$
w_{r \phi}=\frac{3}{2} \rho \Omega \nu ; \quad \nu=\alpha c_{s}^{2} / \Omega,
$$

where $c_{s} \equiv$ sound speed, $\nu$ is the "anomalous viscosity" and $\alpha$ its nondimensional counterpart. This equation is not fundamental; it is the simplest possible representation of the effects of turbulence with the correct dimensional form.

So perhaps if we cannot, god-like, simulate an entire active galactic nucleus on the computer, we can at least set the more modest goal of using numerical experiments to understand the origin and evolution of $w_{r \phi}$.

The turbulent shear stress, or "anomalous viscosity," has accreted an aura of mystery over the years that is not entirely deserved. We know what the basic governing equations are. They do not involve exotic particle physics. In many cases (ADAFs and disk coronae are potentially important exceptions) they do not even involve plasma kinetics. One may simply treat the disk as a magnetized fluid. Then

$$
\frac{D \mathbf{v}}{D t}=-\frac{\nabla p}{\rho}-\frac{\nabla B^{2}}{8 \pi \rho}+\frac{(\mathbf{B} \cdot \nabla) \mathbf{B}}{4 \pi \rho}-\nabla \phi,
$$

where $\phi \equiv$ gravitational potential. Any change in the angular momentum of the fluid in the disk is due to a torque $\mathbf{N}=\mathbf{r} \times D \mathbf{v} / D t$. Torques can only be due, then, to pressure gradients, magnetic forces, or nonaxisymmetric gravitational fields. Radiation forces, which have been dropped here, are negligible except in certain special circumstances.

The fluid equations are easy to write down but hard to solve. To make a numerical solution practical, one wants to (1) include the minimal relevant physics, (2) take advantage of a symmetry, such as axisymmetry, if possible, and (3) start with a simple disk model.

The first of these considerations motivated early workers on disk dynamics to neglect magnetic forces in the equation of motion. Since the discovery by Balbus \& Hawley [4] of a linear instability in weakly magnetized disks, it has become clear 
that this approximation, while nobly motivated, misses perhaps the most important physics (some disks, however, may be so neutral that they are decoupled from the magnetic field $[5,6])$.

One might still hope to use axisymmetry. Alas, early axisymmetric models of magnetized disks showed the development of structures called "channel solutions," consisting of superposed layers of material with large radial velocities [7]. An analytical study [8] showed that these structures are unstable in three dimensions. The upshot is that two dimensional experiments have a nonlinear outcome that is completely different from that of three dimensional experiments. While it is possible that channel solutions may be relevant under certain special conditions, three dimensional experiments are really required to advance our understanding of disks.

Finally, one wants to start with a simple disk model. A natural choice is the "local model," which is a rigorous first order expansion of the equations of motion in $\epsilon=H(r) / r \ll 1(H(r) \equiv$ disk scale height) in a frame comoving with the disk. Boundary conditions are also needed so that the local model can be mapped to a finite computational domain. The "shearing box" boundary conditions [9-11] are well suited to this. They are similar to periodic boundary conditions except that they allow for the presence of shear due to differential rotation. They permit the study of a small piece of the disk without reference to poorly understood inner and outer radial boundaries, although vertical boundary conditions must still be supplied.

All this motivates interest in numerical experiments in the shearing box. I will review recent work, then discuss open questions that might be addressed with more work, and more CPU cycles, in the future.

\section{NUMERICAL EXPERIMENTS AND RESULTS}

The 3D shearing box experiments done to date have all considered a compressible fluid using finite difference or finite-difference-like methods ${ }^{1}$ They have included a variety of physics: magnetic fields and pure hydrodynamics; resistive [12] and ambipolar [14] diffusion; other-than-Keplerian rotation curves [15]; forced convection from heating at the midplane [16,17]; and self-gravity [18].

Shearing box experiments may be divided into two broad classes. The first class are unstratified, i.e. neglect the vertical structure of the disk. Thus if $\phi$ is the gravitational potential, $d \phi / d z=0$ and so in the initial laminar equilibrium $d \rho / d z=$ 0 . The second class are stratified. They include the usual vertical tidal potential $\phi=\frac{1}{2} \Omega^{2} z^{2}$, so an isothermal equilibrium would have $\rho=\rho_{0} \exp \left(-z^{2} /\left(2 H^{2}\right)\right)$.

1) A incompressible study of MHD turbulence in disks using pseudo-spectral methods is also possible and would provide an interesting check on the numerics. 


\section{Unstratified, Magnetized Shearing Box}

Why not dispense with unstratified models and go straight to the more realistic, stratified case? First, the unstratified models are numerically less demanding. The stratified boxes include low density regions that require small timesteps because of the Courant condition in a magnetized fluid. Stratified boxes also assign only a fraction of the zones to the turbulent center of the disk; the rest are assigned to the nearly force-free disk atmosphere. Second, the unstratified boxes allow the study of the nonlinear development of the Balbus-Hawley instability independent of buoyancy effects. A final, post hoc justification is that stratification is not yet measured to have a significant effect on the outcome. This may, however, not be true in future large, highly resolved experiments.

The model has several important dimensionless parameters. One set of parameters describes the shape and size of the box relative to $H=c_{s} / \Omega$. Typically $L_{r} \times L_{\phi} \times L_{z}=(1 \times 2 \pi \times 1) H$. It is natural to set $L_{z}=(1-2) H$; this simulates the limited vertical scale available in a disk. The other dimensions are limited only by CPU time. Another set of dimensionless parameters describes the numerical resolution. Most experiments to date use $2^{5}$ to $2^{7}$ zones along each axis. A final set of parameters describes the mean magnetic field $\langle\mathbf{B}\rangle$ (the brackets denote a spatial average), made dimensionless by comparison of the Alfvén speed with $c_{s}$. The boundary conditions force $\left\langle B_{z}\right\rangle$ and $\left\langle B_{r}\right\rangle$ to be constant in time, while $\left\langle B_{\phi}\right\rangle$ is fixed if and only if $\left\langle B_{r}\right\rangle=0$.

Two groups have reported unstratified shearing box experiments [11,19,12]. All show the development of MHD turbulence initiated by the Balbus-Hawley instability; the turbulence eventually settles down into a final state that is independent of all the initial conditions except the mean magnetic field.

There are many interesting quantities that can be measured in the final state. I will focus on just one: the shear stress in the nondimensional form

$$
\alpha \equiv \frac{2 w_{r \phi}}{3 \rho c_{s}^{2}}=\frac{2}{3 \rho c_{s}^{2}}\left\langle-\frac{B_{r} B_{\phi}}{4 \pi}+\rho v_{r} \delta v_{\phi}\right\rangle
$$

where I have assumed the disk is Keplerian. The first term is the magnetic, or "Maxwell," stress, and the second term is the fluid, or "Reynolds" stress. The brackets indicate an average in $r, \phi, z$ and $t$.

The single most important result to emerge from the unstratified shearing box experiments is that the Balbus-Hawley instability initiates MHD turbulence that has $\alpha \gtrsim 10^{-2}$. No other proposed mechanism for angular momentum transport (except gravitational instability) has produced this result in a purely local disk model.

Measured values of $\alpha$ depend on the box size, on the mean magnetic field strength, and at worst very weakly on numerical resolution $[11,12]$. Crudely speaking,

$$
\alpha \sim 0.01+4 \frac{\left\langle V_{A, z}\right\rangle}{c_{s}}+\frac{1}{4} \frac{\left\langle V_{A, \phi}\right\rangle}{c_{s}}
$$


where $\left\langle\mathbf{V}_{A}\right\rangle \equiv$ mean Alfvén velocity. Thus for weak mean fields, $\alpha \sim 10^{-2}$, while intermediate strength mean fields raise $\alpha$ above this base level. For mean fields that are strong in the sense that $V_{A, i}$ is large compared to $L_{i} \Omega$ the field is linearly stable and equation (5) does not apply (see $[4,13]$ for linear stability criteria). The flow is laminar, and $\alpha \rightarrow 0$. Equation (5) should not be taken too seriously as yet; while the sense is likely correct, the coefficients could change substantially in future experiments.

Notice that in the absence of a mean field all the currents that sustain the field are contained within the computational domain and so are subject to decay. In this sense, the zero-mean-field experiments demonstrate the existence of a dynamo: they show that MHD turbulence in disks, driven by the Balbus-Hawley instability, can sustain a magnetic field in the presence of dissipation [12].

Finally, some zero-mean-field experiments [12] included a finite resistive diffusivity $\eta$. These show that $\alpha$ is sensitive to the presence of resistive diffusion. Defining the magnetic Reynolds number $R e_{M} \equiv c_{s} H / \eta$, turbulence decays at $R e_{M} \lesssim 10^{3}-10^{4}$. Dwarf novae disks in quiescence may fall in or below this range [6].

\section{Magnetized, Stratified Shearing Box}

Stratified shearing box experiments have been carried out by two groups $[14,20,21,15]$. The experiments have $L_{z}=4-6 \times \sqrt{2} H$, and numerical resolution similar to the unstratified models. Both groups report generally consistent results, which is remarkable since very different numerical methods were used. The stratified experiments give $\alpha \sim 10^{-2.5}$.

The stratified boxes produce $\alpha$ slightly lower than the unstratified boxes. Part or all of this difference may be due to the lower effective numerical resolution of the more numerically demanding stratified experiments. The stratified boxes are sensitive to resolution in that, when the resolution is increased, $\alpha$ also increases $[21,20]$. Once higher numerical resolution becomes practical, the stratified experiments ought to be repeated until convergence can be demonstrated.

The measured $\alpha$ is insensitive to the vertical boundary conditions. Again, this may change in larger, converged experiments. Some experiments have used vertical boundary conditions that allow the mean field to evolve $[14,20,15]$. In my view this is risky because the mean field is generated by currents "in the boundary" and not within the computational volume itself; changes in the mean field are thus generated by interaction of the fluid with the boundary conditions. This is, however, mainly a matter of taste. It is fair to say that we do not yet know what the most relevant vertical boundary conditions are.

One of the main motivations for the stratified experiments is that they can in principle be used to estimate the vertical run of turbulent dissipation in disks, thereby removing a serious obstacle to predictive models for disk spectra. In isothermal models the run of dissipation is not calculated directly, but two closely related 
quantities are: $w_{r \phi}(z)$ and $S_{z}(z)$, the vertical component of the Poynting flux. It is found that [21]

$$
S_{z}(z=H) \simeq 0.01 \int_{0}^{H} d z \frac{3}{2} \Omega w_{r \phi}(z)
$$

Thus only a small fraction of the power extracted by the turbulent shear stress from the differential rotation emerges as MHD waves. Larger values of $\alpha$ in future experiments, however, would imply stronger fields and hence greater magnetic buoyancy. It is also found [21] that $\alpha$ is not constant with height, as is commonly assumed. A better fit to the data is given by $\alpha \sim\left(\rho / \rho_{0}\right)^{-1 / 2}$, consistent with equation (5). Clearly the situation with the stratified experiments is not entirely satisfactory; it is a challenge for future experiments to make the dissipation and vertical energy transport explicit in a converged calculation.

\section{Unmagnetized Box}

Are there any local transport processes that can compete with or dominate MHD turbulence? And what transport processes govern the evolution of disks that are nearly neutral and thus poorly coupled to the magnetic field?

Convectively driven turbulence was once thought a promising transport mechanism $[22,23]$. There was an early warning that something might be amiss, however, from a quasilinear study [24] which showed that nonaxisymmetric convective modes produce an inward angular momentum flux. Subsequent shearing box experiments $[16,17]$ showed that both forced convection and overturning of an initially unstably stratified disk led to inward angular momentum transport $(\alpha<0)$ in the fully nonlinear regime. This counterintuitive result is a nice illustration of the value of numerical experiments.

There is still one regime in which convectively driven angular momentum transport could play a role. That is in geometrically thick flows with unstable radial stratification- for example ADAFs (see [25] for a review). A shearing box experiment with forced radial convection might be revealing, but the shearing box is not a good model for these flows. Global models are really required.

Shearing box experiments have also permitted the direct evaluation of another once-promising mechanism for initiating turbulence in disks: nonlinear hydrodynamic instability [26]. In these experiments a purely hydrodynamical Keplerian shear flow is violently perturbed. It is found that the flow returns to a laminar state, independent of the amplitude of the initial perturbation.

Because the numerical experiments are run at a Reynolds number that is low in comparison to that in astrophysical disks, nonlinear hydrodynamic instability cannot be rigorously ruled out. But analytic arguments [26] (see also Balbus, this volume) and the absence of nonlinear instability in laboratory analogs relevant to Keplerian shear flow (see [27] and references therein) make it likely, in my view, that Keplerian disks are nonlinearly hydrodynamically stable. 
Finally, self-gravity may be noticeable or even dominant in disks around young stars and in active galactic nuclei. The shearing box has been used to study gravitational instability in cooling disks [18]. Cooling is essential since in its absence the disk simply heats up until it is stable. The outcome is a fluctuating state with Toomre's stability parameter $\langle Q\rangle \sim 1$ and significant outward transport of angular momentum via gravitational and Reynolds stresses. These experiments also show that self-gravity produces truly local transport in a sense to be made clear below.

\section{OPEN QUESTIONS}

I will conclude with a short list of open questions that are astronomically relevant and can be answered in the near future with numerical experiments in the shearing box.

1. Is $\alpha$ determined locally? A different way of phrasing this question is, does $\alpha$ converge as the planar box size $L_{r}$ and $L_{\phi}$ are increased? Experiments to date find turbulence with most of the energy in structures with scale comparable to the box size. Thus the outcome is limited by the box size.

If $\alpha$ is determined locally one expects that the autocorrelation function of fluid variables to decay rapidly on scales $\gtrsim H$. Equivalently, fluid variable power spectra should turn over and decline at small $k_{r}$ and $k_{\phi}$. Because the natural scale for the turnover is $H$, it should be seen in boxes only slightly larger than current experiments. The detection of the turnover would be a significant milestone for disk physics in that it would show that $\alpha$ is locally determined and so validate the use of the local model.

The alternative is that the fluid autocorrelation functions decline only slowly or not at all with distance in the plane of the disk. Then the shear stress at any point in the disk can be influenced by conditions at points many scale heights away. This is not inconceivable. The $\alpha \sim(H / r)^{n}$ prescription common in studies of dwarf novae, for example, requires this sort of nonlocality because local turbulence must somehow "know" about the large scale structure of the disk and in particular the local radius $r$. If $\alpha$ is nonlocal, however, we must abandon the shearing box and move to global models. In what follows I assume that $\alpha$ is local and thus that the shearing box is relevant.

2. How does $\alpha$ depend on resistive diffusion $\eta$, viscosity $\nu$, and their ratio, the magnetic Prandtl number $\operatorname{Pr}_{M} \equiv \nu / \eta$ ? In most circumstances both resistivity and viscosity are negligible, but even then, it has been argued, $P r_{M}$ may govern the character of MHD turbulence (see [27] and references therein). This hypothesis can be tested with sufficiently high resolution experiments that allow a reasonable separation of the resistive and viscous lengthscales. Resistivity is not always negligible, however. Recent work [6] shows that the standard disk instability model for dwarf novae implies a magnetic Reynolds number of order $10^{3}$ for dwarf nova disks in quiescence. Such low $R e_{M}$ may have a direct impact on the development of MHD turbulence (cf. [12]). Protostellar disks also suffer from high resistivity; some 
parts are likely to be completely decoupled from the magnetic field [5]. This issue has direct astronomical relevance and can be studied with codes and computers that are now available.

3. How does $\alpha$ vary in time? Local model MHD experiments have so far only sought to measure $\alpha$ when the disk scale height is steady in time. Some of the most potentially revealing phenomena in disk systems, however, involve rapid changes in disk temperature. Under these circumstances, how long does it take $\alpha$ to readjust to its steady state value? There is already preliminary evidence that this relaxation time is long in that the time required for the zero-mean-field unstratified experiments to reach a steady state is many orbital periods [12]. But a more direct determination can be made in stratified, isothermal shearing box experiments in which the temperature is forced to change suddenly. If the relaxation time is long compared to the thermal timescale, then the structure of cooling and heating fronts in dwarf nova disks may be quite different from what is now imagined.

4. How does a vary near disk edges? Gaps may be opened in disks around young stellar objects by stellar or planetary companions. The size of the gap and accompanying torque on the companion are sensitive to the surface density profile close to the disk edge, which is determined by a balance between tidal torques on the disk and all other torques. One way of studying disk edges would be to allow a point mass to orbit in the shearing box and clear a gap in the disk. Such an experiment could test whether $\alpha$ varies near the edge of the disk in a way that is consistent with the usual treatment of $w_{r \phi}$ as a viscous stress. Perhaps it does not; perhaps $\alpha$ increases rapidly within a few $\times 2 \pi H$ of the edge. Such experiments could also test whether magnetic fields drive cross-gap accretion. Numerical work to date has focused on the nonmagnetic problem (e.g. [28]), but this approximation may be missing an important piece of the puzzle.

\section{ACKNOWLEDGEMENTS}

I am grateful to Steve Balbus, John Hawley, Kristen Menou, Eve Ostriker, Eliot Quataert, and Jim Stone for their comments. This work was supported by NASA grant NAG 52837.

\section{REFERENCES}

1. Ostriker, J. P. 1985, in The Milky Way Galaxy, eds. H. Van Woerden, R. J. Allen, \& W. B. Burton (Dordrecht: Reidel) 635

2. Cannizzo, J. 1993, in Accretion Disks in Compact Stellar Systems ed. J. Wheeler (Singapore: World Scientific), 6

3. Hameury, J.-M. et al. 1998, in preparation

4. Balbus, S.A., \& Hawley, J.F. 1991, ApJ, 376, 214

5. Gammie, C.F. 1996, ApJ, 457, 355

6. Gammie, C.F., \& Menou, K. 1998, ApJ, 492, L75 
7. Hawley, J.F., \& Balbus, S.A. 1992, ApJ, 400, 595

8. Goodman, J., \& Xu, G. 1994, ApJ, 432, 213

9. Toomre, A. 1981 in The Structure and Evolution of Normal Galaxies, eds. S.M. Fall \& D. Lynden-Bell (Cambridge: Cambridge University Press), 111

10. Wisdom, J., \& Tremaine, S. 1988, AJ, 95, 925

11. Hawley, J.F., Gammie, C.F., \& Balbus, S.A. 1995, ApJ, 440, 742

12. Hawley, J.F., Gammie, C.F., \& Balbus, S.A. 1996, ApJ, 464, 690

13. Balbus, S.A., \& Hawley, J.F. 1992, ApJ, 400, 610

14. Brandenburg, A., Nordlund, A., Stein, R.F., \& Torkelsson, U. 1995, ApJ, 446, 741

15. Abramowicz, M., Brandenburg, A., \& Lasota, J.-P. 1996, MNRAS, 281, L21

16. Stone, J.M., \& Balbus, S.A. 1996, ApJ, 464, 364

17. Cabot, W. 1996, ApJ, 465, 874

18. Gammie, C.F. 1998, in preparation

19. Matsumoto, R., \& Tajima, T. 1995, ApJ, 445, 767

20. Brandenburg, A., Nordlund, A., Stein, R.F., \& Torkelsson, U. 1996, ApJ, 458, L45

21. Stone, J.M., Hawley, J.F., Gammie, C.F., \& Balbus, S.A. 1996, ApJ, 463, 656

22. Cameron, A.G.W. 1978, Moon Planets 18, 5

23. Lin, D.N.C., \& Papaloizou, J.C.B. 1980, MNRAS, 191, 37

24. Ryu, D. \& Goodman, J. 1992, ApJ, 388, 438

25. Narayan, R., Mahadevan, R., \& Quataert, E. 1997, in Proc. Reykjavik Symp. on Non-linear Phenomena in Accretion Disks Around Black Holes, eds. M.A. Abramowicz, G. Bjornsson, \& J.E. Pringle, in press

26. Balbus, S.A., Hawley, J.F., \& Stone, J.M. 1996, ApJ, 467, 76

27. Balbus, S.A., \& Hawley, J.F. 1998, Rev. Mod. Phys., in press

28. Artymowicz, P., \& Lubow, S.H. 1996, ApJ, 467, L77 